\title{
Discussion: Treatment of psoriatic arthritis and psoriasis
}

\author{
P J Mease with contributions from W-H Boehncke, D D Gladman, A Marchesoni, A Menter
}

Ann Rheum Dis 2005;64(Suppl II):ii91-ii92. doi: 10.1136/ard.2004.030940

\begin{abstract}
Why does it occasionally happen, with some medications and individuals, that there is a significant effect of treatment on the joints but not the skin, and vice versa?

Mease: Although most often we see a significant correlation between joint and skin response with current systemic treatments, it is true that there is sometimes a disconnect. A variety of factors lie behind this phenomenon. Even though cellular and cytokine activation profiles are very similar in the joint and the skin, there are still some important differences. Some key cellular elements present in the joint, such as fibroblasts, chondrocytes, and osteoclasts are not present or have a lesser role in the skin, just as keratinocytes are not active in joints. There are likely subtly different quantities of cytokines in the two compartments. Vascularisation may be different; drug penetrance of the compartment may be different. Thus, it stands to reason that there may be differential sensitivities to medication effect.
\end{abstract}

\section{Why is there such a discrepancy between dermatologists' and rheumatologists' recommendations regarding periodic liver biopsies to monitor the hepatic safety of methotrexate?}

Mease: It is not uncommon to observe a dermatologist recommending liver biopsy, especially in the USA, to monitor the effects of methotrexate after every $1500 \mathrm{mg}$ of use. Rheumatologists virtually never do so, unless there is a specific indication, such as persistently elevated liver function tests or other signs of liver toxicity. Part of this stems from case series of psoriasis patients, in whom there appears to be a greater chance for progression from one stage of hepatotoxicity to the next when serial liver biopsy studies are done in patients on methotrexate, as distinct from studies of patients with rheumatoid arthritis (RA), in whom progression of liver grade is much less common. It is not entirely clear whether this discrepancy is related to lack of comparability of studies or differences in potential for hepatic toxicity related to the underlying diseases. Because of the concern about methotrexate hepatotoxicity in patients with psoriasis, there may be a greater interest in using antitumour necrosis factor (anti-TNF) agents and other biologicals as monotherapy, rather than in combination with methotrexate, as has been the trend in RA therapy.

Menter: The guidelines about monitoring psoriasis patients on methotrexate therapy are quite clear, recently updated in 1998, and based on 30+ years of experience with use of the drug, unlike rheumatology guidelines, which are based on a shorter experience and are quite different. Dermatologists take these guidelines seriously, given the experience on which they are based.

Gladman: One must remember that the original experience in dermatology was with a different dose schedule and less stringent patient follow up. The rheumatology guidelines are based on extensive experience in RA, not on information collected from patients with psoriatic arthritis (PsA).

Menter: Do similar principles regarding differential effects of methotrexate and anti-TNF agents apply in PsA as in RA: do both yield improvement of symptoms and signs in the majority of patients, whereas anti-TNF medications are much more effective in slowing or halting disease progression?

Mease: Much less is known about this point in PsA, compared with what we know in RA. There has been no head to head trial of methotrexate versus anti-TNF, and methotrexate has been inadequately studied in PsA. What we do know is that in patients responding inadequately to methotrexate, addition of anti-TNF medication significantly improves signs and symptoms and in the one trial with radiologic results, the etanercept group experienced a slowing or halting of radiologic progression over two years of treatment. A retrospective analysis of patients treated with methotrexate by Gladman's group suggested that these patients progressed radiologically similar to a non-methotrexate treated matched cohort of patients with PsA, but this type of analysis is admittedly fraught with problems. More data are needed to truly know what methotrexate can accomplish in this arena.

Marchesoni: Whether patients with PsA are more likely to develop methotrexate liver toxicity than RA patients is an old issue. Some years ago, in a small study, we did not find any relevant difference in liver toxicity between RA and PsA patients treated with methotrexate. However, most rheumatologists feel that discontinuation of methotrexate because of elevated liver function tests is much more common in patients with PsA. Regardless of the percentage of PsA patients discontinuing methotrexate therapy because of transaminase abnormalities, the risk of severe liver damage in these patients does not seem to be higher than in RA patients. Therefore, there is no real need for liver biopsy in PsA patients treated with methotrexate, at least no more than in patients with RA. In Europe too, dermatologists are very concerned about methotrexate use presumably because historically they started to use methotrexate many years before the rheumatologists did and, at that time, methotrexate had been used only at high doses as chemotherapy for cancer. It does not sound logical not to use methotrexate in combination with anti-TNF agents just because of the concern about liver toxicity.

Do patients with psoriasis and PsA have unique safety and tolerability problems with anti-TNF agents and other biologicals?

Mease: To date, no unique safety and tolerability issues have arisen in trials of psoriasis and PsA compared with trials in RA, ankylosing spondylitis, and other inflammatory diseases.

Abbreviations: DMARD, disease modifying antirheumatic drug; $\mathrm{Ps}_{\mathrm{s}}$, psoriatic arthritis; RA, rheumatoid arthritis; TNF, tumour necrosis factor 
Specifically, there has not been a problem with Koebner's phenomenon in those patients using subcutaneously administered medications. There has not been an increased incidence of cellulitis or other infections that could arise from immunosuppression, such as herpes simplex, despite the lack of barrier integrity of lesional skin and immunosuppressive capability of the medications. Although there have been reports of squamous cell carcinoma in psoriasis patients treated with anti-TNF medications, the numbers of these patients have not risen above what is expected in the psoriasis population-bearing in mind that some of these patients have received previous PUVA therapy, which predisposes patients in this regard. In general, trials have not been large enough to witness some of the adverse events that have been observed in post-marketing surveillance of large numbers of patients treated with the anti-TNF agents for RA, for example, but there is no reason to think that these patients will escape some of these side effects as commercial use expands.

Marchesoni: Although there are no specific studies about the effects of the traditional disease modifying antirheumatic drugs (DMARDs) in preventing irreversible joint damage, indirect evidence suggests that anti-TNF agents are much more effective in this respect. This raises the same issues as in RA: Should we use aggressive therapy in early disease in patients at risk of bad outcomes? If so, which kind of aggressive therapy-DMARDs in combination, anti-TNF alone, anti-TNF in combination with DMARDs? This leads to the hot issue raised by Gladman: When should anti-TNF therapy be started in PsA? In my opinion, it should be started in the following situations: patients with active disease despite appropriate DMARD therapy (that is, at least two DMARDs, one of which should be methotrexate and the other sulfasalazine, ciclosporin A, or leflunomide, first alone and then in combination); patients showing rapid radiographic deterioration; patients with severe axial involvement refractory to non-steroidal anti-inflammatory drugs (NSAIDs); and patients with severe skin involvement refractory to conventional therapy (PUVA, ciclosporin A, methotrexate). In addition, we should evaluate very early therapy in patients with potentially aggressive joint disease (induction and then maintenance with DMARDs) and cyclic therapy to treat only joint and/or skin relapses because PsA may have a cyclic course. The extent of joint involvement is obviously important when deciding the therapy, although in PsA even a single joint, such as a swollen knee, should be considered enough to start anti-TNF therapy if the patient is not responding to other treatments and is disabled by it. Guidelines for the use of anti-TNF agents will be provided by the national health systems of countries, but I am afraid that they will be strongly influenced by economic factors (at least in Italy). The safety profile of the anti-TNFs in PsA should be comparable with that of other rheumatic conditions. Currently there is no real reason to think that this therapy should be more toxic in PsA.

Boehncke: In this context, one may point out the impact of skin involvement on this decision: active and widespread cutaneous involvement would ask for earlier use of biologicals (at least of those that have known efficacy in both skin and joint compartments).

\section{What about use of ciclosporin in conjunction with the newer biological agents?}

Mease: Ciclosporin used in combination with methotrexate has been shown to be more effective than either drug alone in RA and more recently, in PsA, but in general, ciclosporin is not used widely by rheumatologists because of relatively low therapeutic effect, especially in the context of potential safety issues with the drug. However, it has been shown to be very effective in quickly improving psoriasis lesions. Although its combination with biological agents has not yet been formally tested for safety and efficacy, an appealing hypothesis is that such use-that is, ciclosporin in combination with biologicals, may augment the rapidity of response and degree of efficacy in controlling the skin lesions of psoriasis, even while the majority of the arthritis response appears to be accomplished by the biological agent. It is also conceivable that a lower dose and possibly briefer dose duration may be adequate to achieve such an effect, thus lowering the toxicity potential.

Menter: In clinical practice, although not in clinical trials, ciclosporin has been overlapped for approximately eight weeks with introduction of biological therapy, and then tapered off, partly to help achieve a more rapid and effective clearing of skin with maintenance of clinical response.

\section{How important is it to treat entheseal inflammation in PsA?}

Mease: There is an increasing interest in the enthesium, the location of tendon and ligament insertion into bone, as a source of symptom burden in patients with PsA. The transgenic rat model of spondyloarthropathy shows evidence of entheseal inflammation well before synovitis sets in. Many patients with PsA describe tenderness and stiffness in areas apart from the joint per se, and many do not display much burden of true synovitis. Indeed, older studies which suggested a lower prevalence of PsA in patients with psoriasis may have mislabelled PsA patients with primarily entheseal inflammation. The response of entheseal inflammation to medical treatment has virtually never been studied due to the dearth of the use of valid and feasible outcome measures. An exploratory assessment of entheseal response was done in the phase II trial of infliximab in PsA and such assessments have been included in subsequent trials. These demonstrate a good clinical response to at least anti-TNF medications. Although it is unclear if clearing of enthesopathy will have an impact on disease progression, it does appear to reduce symptom burden.

Marchesoni: It is true that in PsA entheseal inflammation is still underestimated. Its treatment should be evaluated more carefully, also for pathogenetic reasons.

Correspondence to: Dr P J Mease, Seattle Rheumatology Associates, 1101 Madison, Suite 230, Seattle, WA 98104, USA; pmease@nwlink. com 